\title{
Informed Consent for Obstetrics Management: A Urogynecologic Perspective
}

\author{
G. W. Davila \\ Cleveland Clinic Florida, Fort Lauderdale, Florida, USA
}

A recent editorial by Dr W. Benson Harer titled 'Patient choice cesarean' in the March/April 2000 ACOG Clinical Review is of great interest to practicing obstetricians/gynecologists, and in particular to urogynecologists [1]. I met Dr Harer (currently President of the American College of Obstetricians and Gynecologists) after my presentation at the IUGA-sponsored Pelvic Floor Dysfunctions symposium at last year's FIGO meeting in Washington, DC, and discussed the growing interest in the concept of patient informed consent for obstetric management at term. This is an issue which is beginning to be addressed in the United States. In many other countries pregnant women have decision-making power regarding the management of their labors. As clinicians who treat women suffering from many serious acute and long-term consequences of vaginal birth, urogynecologists are in a unique position to bring a more objective and scientific viewpoint to the importance of allowing pregnant women (and their spouses) to participate in making informed decisions regarding their obstetric management.

\section{Maternal and Fetal Consequences of Vaginal Birth}

Adverse consequences to the mother as well as the newborn can result from a vaginal delivery. The medical literature is replete with information regarding short- as well as long-term obstetrical consequences and their expected incidence. It is well known, and increasingly

Correspondence and offprint requests to: Dr G. W. Davila, Department of Gynecology, Section of Urogynecology and Reconstructive Pelvic Surgery, Cleveland Clinic Florida, 3000 West Cypress Creek Road, Fort Lauderdale, Florida 33309, USA. Tel: 954-9785059; Fax: 954-978-5892; Email: davilag@ccf.org understood, that urinary incontinence and genital prolapse may affect more than $25 \%$ of adult women, with significant adverse quality of life implications. Pudendal nerve damage and direct soft tissue trauma during the process of vaginal delivery has been demonstrated to be of etiologic importance in the development of urinary and fecal incontinence [2]. The levator musculature is injured during the birth process, resulting in significant impairment of pelvic floor muscular strength when compared to elective cesarean delivery [3]. In addition, both overt and occult anal sphincter trauma occurring during a vaginal delivery may result in the development of fecal incontinence [4]. Anal incontinence occurs in a large number of middle-aged women, the risk being increased by the previous occurrence of a vaginal delivery [5]. Vaginal birth is also associated with the development of genital prolapse and the associated need for reconstructive surgery [6]. Neurophysiologic studies have demonstrated the etiologic role of parturition-related nerve damage in the development of pelvic floor dysfunction [7]. Recently, intrapartum monitoring of pudendal nerve function has been shown to be feasible and may provide insight into the effects of the delivery process on the maternal pelvic floor [8]. Most symptomatic women improve as healing from the delivery process occurs. However, aging, further obstetric trauma and menopausal hypoestrogenism contribute to the resurgence of symptomatic incontinence and prolapse in future years. Urogynecologists and pelvic reconstructive surgeons are very familiar with the surgical interventions required to restore normal pelvic floor anatomy. It is not widely known, however, that pelvic floor damage can be reduced by minimizing forceps deliveries and episiotomies, by allowing passive descent in the second stage, and by selectively recommending elective cesarean delivery [9]. 
Changes in sexual function, most with a negative impact, are frequently described as resulting from the delivery process. These factors have been less widely studied, although sexual problems, including dyspareunia and decreased satisfaction, are frequently reported by women delivered over an intact perineum as well as in those with an episiotomy [10].

Fetal injury can also result from parturition. Well described potentially serious consequences include brachial plexus injuries, head trauma and labor-related fetal distress. The marked reduction in vaginal breech deliveries represents a clear example of a management alteration to optimize outcomes. Recent studies have demonstrated a significantly lower neonatal morbidity rate with elective cesarean section than with attempted vaginal breech delivery [11]. As with a vertex-presenting fetus, once expulsion has begun it may not be possible to prevent serious fetal injury. No accurate screening tool exists to identify those labors that will result in a difficult delivery or the need for instrumentation.

Cesarean section is a safe operation. There are no recent series reporting the risks associated with a completely elective, scheduled cesarean delivery. During the $1980 \mathrm{~s}$, morbidity rates for routine vaginal and elective cesarean delivery were estimated at $3.7 \%$ and $4.7 \%$, respectively [12]. With current techniques for assessing fetal age, iatrogenic prematurity can be avoided. Routine usage of prophylactic antibiotics, regional anesthesia and a fasting state also minimize potential associated morbidity.

More research is clearly needed in the area of obstetric risk assessment in order to better understand the identification of obstetrical trauma risk factors.

\section{Why Informed Consent?}

Obtaining informed consent prior to the onset of labor is at the crux of the matter. It must be assumed that providing pregnant women with information regarding the risks and benefits of the vaginal birth process will result in an increased cesarean section rate. The typical young urogynecologic patient seen with a labor-related complaint such as fecal incontinence is quick to point out that 'I never knew this could happen'. As many consequences of the vaginal delivery process occur with a predictable frequency, patients can and should be made aware of these potential risks.

As a matter of clinical practice, a patient's informed consent is obtained prior to any invasive procedure associated with known risks and benefits [13]. From minor office procedures to major surgical interventions, the signing of the informed consent form is an accepted and required ritual. Occasionally, this process takes more time than the procedure itself. It would seem rational, as such, that obstetric management informed consent be obtained. Most obstetricians restrict labor-planning discussion with their patients to pain management and the 'birth plan'. In the recent past the routine performance of an episiotomy at delivery received much attention, and its risks and benefits were openly discussed. Obtaining informed consent for the performance of episiotomy has been encouraged [14]. An objective discussion regarding the risks and benefits of the entire labor and delivery process, as well as elective cesarean delivery, would allow patients a clearer understanding of the actual delivery process and to make informed decisions regarding their individual management. ACOG should take the initiative in the development of an unbiased, clearly understandable consent document, once this concept becomes more definite.

Most policy recommendations regarding the US cesarean section rate have focused on fetal wellbeing, and recommendations have been made to reduce rates to proposed benchmarks [15]. As many of the common consequences of the vaginal birth process can have a markedly negative impact on the quality of life of the mother, it is incumbent upon the clinician to discuss these issues at a time remote from the actual birth process.

\section{Cultural Variations and Elective Cesarean Section}

In Brazil and other Latin American countries, cesarean section on demand is well accepted by society and by practicing obstetricians [1] (personal communication). Potential complications associated with cesarean delivery are minimized by the elective, scheduled performance of the operation. In these cultures women have accepted elective, scheduled cesarean as a safe means of avoiding pelvic floor damage and its associated consequences of urinary, fecal and sexual dysfunction. Such 'perineal prophylaxis' is valued by both clinicians and patients as an actual improvement in obstetric care.

The Brazilian experience has demonstrated that a high controlled, scheduled cesarean delivery rate is associated with minimal maternal and fetal morbidity. If morbidity is used as a primary outcome measure, it is highly likely that a comparative series would show that a high cesarean delivery rate is superior to a high vaginal delivery rate. The highest-risk cesarean delivery is the unplanned operation performed for failed labor or fetal distress.

In the US, an obstetrician's cesarean section rate has been used by insurance companies and hospitals as a measure of the quality of care provided. As a multitude of factors affect a clinician's operative delivery rate, this single statistic has received much criticism from clinicians. However, ACOG and other organizations continue to look at a clinician's cesarean delivery rate as being continuously reducible. The cesarean section rate should not be used as an indicator of quality of obstetrical care. As the concept of elective cesarean grows in popularity on the US, this statistic will be minimized in value. 


\section{The Cost Issues}

Currently, most insurance companies in the US reimburse equally for vaginal or cesarean delivery. There is thus no financial incentive for obstetricians to perform more cesarean sections. In a theoretical model analyzing a $100 \%$ scheduled cesarean delivery rate, the calculated financial costs were found to be significantly lower than those associated with a high vaginal delivery rate, on a short- as well as a long-term basis [16]. Scheduled elective cesarean delivery obviates various significant costs incurred during the labor process. Labor room and nursing costs are minimized, especially compared to long and/or induced labors. Early hospital discharge, as is used for hysterectomy procedures, minimizes postoperative costs. Financial factors support an increasing cesarean delivery rate.

\section{Why Should Urogynecologists Care?}

As pelvic floor medicine specialists, our waiting rooms are filled with women with urinary and fecal incontinence, genital prolapse, pelvic pain and other conditions well known to be etiologically related to previous vaginal deliveries. We see the daily hygienic and social impacts of pelvic floor dysfunction. Our current treatment options for pelvic floor dysfunction are quite good, but have not achieved (and will not achieve) a $100 \%$ cure rate for any or all pelvic floor problems. Although a high cesarean section rate would undoubteldy have a negative patient volume and financial impact on urogynecologic practice, we cannot deny the facts: many of the medical problems we care for on a daily basis are preventable. We cannot go as far as encouraging cesarean delivery for all; however, we should firmly encourage patient education regarding obstetric consequences and management options - which is, in fact, the informed consent process.

\section{Summary}

Obstetric management has been modified for conditions with acute, short-term consequences (i.e. breech presentation). It is timely to address those conditions related to the vaginal birth process which may have a delayed but significant negative impact on the mother's quality of life (i.e. urinary and fecal incontinence) but which may be reduced by selectively avoiding the vaginal birth process. The known possible consequences, and other associated risks and benefits, should be discussed with the pregnant patient and her spouse, in order to allow their input into the obstetric decision-making process and to individualize management. Urogynecologists are in a unique, no-conflict-of-interest position to further the acceptance of the concept of obstetrical informed consent. Cesarean delivery should not be seen as a failure of obstetric management, but rather as a safe and acceptable option to the vaginal delivery process, chosen after completing an informed decision-making process.

\section{References}

1. Harer WB. Patient choice cesarean. ACOG Clin Rev 2000;5:1

2. Meyer S, Schreyer A, De Grandi P, Hohlfeld P. The effects of birth on urinary continence mechanisms and other pelvic-floor characteristics. Obstet Gynecol 1998;92:613-618

3. Peschers UM, Schaer GN, DeLancey JO, Schuessler B. Levator ani function before and after childbirth. Br J Obstet Gynaecol 1997; 104:1004-1008

4. Sultan AH, Kamm MA, Hudson CN, Thomas JM, Bartram CI. Anal sphincter disruption during vaginal delivery. $N$ Engl J Med 1993;329:1905-1911

5. Nygaard IE, Rao SS, Dawson JD. Anal incontinence after anal sphincter disruption: a 30-year retrospective cohort study. Obstet Gynecol 1997;89:896-901

6. Mant J, Painter R, Vessey M. Epidemiology of genital prolapse: observations from the Oxford Family Planning Association Study. Br J Obstet Gynaecol 1997;104:579-585

7. Snooks SJ, Swash M, Mathers SE, Henry MM. Effect of vaginal delivery on the pelvic floor: a 5-year follow-up. $\mathrm{Br} J$ Surg 1990;77:1358-1360

8. Clark MH, Scott M, Vogt V, Benson JT. Monitoring pudendal nerve function during labor. Obstet Gynecol 2001;97:637-639

9. Handa VL, Harris TA, Ostergard DR. Protecting the pelvic floor: obstetric management to prevent incontinence and pelvic organ prolapse. Obstet Gynecol 1996;88:470-481

10. Klein MC, Gauthier RJ, Robbins JM et al. Relationships of episiotomy to perineal trauma and morbidity, sexual dysfunction, and pelvic floor relaxation. Am J Obstet Gynecol 1994;171:591598

11. Hannah ME et al. Planned cesarean section versus planned vaginal birth for breech presentation at term: a randomized multicenter trial. Lancet 2000;356:1375-1383

12. Nielsen TF, Hokegard KH. Postoperative cesarean section morbidity: a prospective study. Am J Obstet Gynecol 1983;146:911-915

13. American College of Obstetricians and Gynecologists. Ethical dimensions of informed consent. ACOG Committee Opinion 108. Washington, DC 1992

14. Thorp JM. Patient autonomy, informed consent, and routine episiotomy. Contemp Obstet Gynecol 1995:92-101

15. American College of Obstetricians and Gynecologists. ACOG executive summary evaluation of cesarean delivery. ACOG, Washington, DC, 2000

16. Gottesfeld S, Davila G. Cesarean section: the case for its cost effectiveness. In: Eiseman B and Stahlgren L, eds. Cost effective surgical management. Philadelphia: WB Saunders, 1987:253-255 